\title{
BMJ Open Copattern of depression and alcohol use in medical care patients: cross-sectional study in Germany
}

Diana Guertler (DD , ${ }^{1,2}$ Anne Moehring, ${ }^{1,2}$ Kristian Krause, ${ }^{1}$ Anil Batra, ${ }^{3}$ Sandra Eck, ${ }^{3}$ Jennis Freyer-Adam, ${ }^{2,4}$ Sabina Ulbricht, ${ }^{1,2}$ Hans-Jürgen Rumpf, ${ }^{5}$ Gallus Bischof, ${ }^{5}$ Ulrich John, ${ }^{1,2}$ Christian Meyer ${ }^{1,2}$

To cite: Guertler D,

Moehring A, Krause K, et al. Copattern of depression and alcohol use in medical care patients: cross-sectional study in Germany. BMJ Open 2020;10:e032826. doi:10.1136/ bmjopen-2019-032826

- Prepublication history and additional material for this paper are available online. To view these files, please visit the journal online (http://dx.doi. org/10.1136/bmjopen-2019032826).

Received 08 July 2019 Revised 13 February 2020 Accepted 06 April 2020

\section{Check for updates}

(C) Author(s) (or their employer(s)) 2020. Re-use permitted under CC BY-NC. No commercial re-use. See rights and permissions. Published by BMJ.

For numbered affiliations see end of article.

Correspondence to Dr Diana Guertler; diana.guertler@med.unigreifswald.de

\section{ABSTRACT}

Objective To predict depressive symptom severity and presence of major depression along the full alcohol use continuum.

Design Cross-sectional study.

Setting Ambulatory practices and general hospitals from three sites in Germany.

Participants Consecutive patients aged 18-64 years were proactively approached for an anonymous health screening (participation rate $=87 \%, \mathrm{~N}=12828$ ). Four continuous alcohol use measures were derived from an expanded Alcohol Use Disorder Identification Test (AUDIT): alcohol consumption in grams per day and occasion, excessive consumption in days per months and the AUDIT sum score. Depressive symptoms were assessed for the worst 2-week period in the last 12 months using the Patient Health Questionnaire (PHQ-8). Negative binomial and logistic regression analyses were used to predict depressive symptom severity (PHQ-8 sum score) and presence of major depression (PHQ-8 sum score $\geq 10$ ) by the alcohol use measures.

Results Analyses revealed that depressive symptom severity and presence of major depression were significantly predicted by all alcohol use measures after controlling for sociodemographics and health behaviours $(p<0.05)$. The relationships were curvilinear: lowest depressive symptom severity and odds of major depression were found for alcohol consumptions of $1.1 \mathrm{~g} /$ day, $10.5 \mathrm{~g} / 0$ ccasion, 1 excessive consumption day/month, and those with an AUDIT score of 2. Higher depressive symptom severity and odds of major depression were found for both abstinence from and higher levels of alcohol consumption. Interaction analyses revealed steeper risk increases in women and younger individuals for most alcohol use measures.

Conclusion Findings indicate that alcohol use and depression in medical care patients are associated in a curvilinear manner and that moderation by gender and age is present.

\section{INTRODUCTION}

Clarification of associations between depression and alcohol use would allow for more effective intervention strategies among comorbid populations. ${ }^{1}$

\section{Strengths and limitations of this study}

- This study's use of multivariable fractional polynomials to systematically model curvilinearity in the association of depression and multiple continuous alcohol use measures is novel and enabled an examination across the full alcohol use continuum, as well as statistical testing for gender and age interactions.

- A large, proactively recruited medical care sample was investigated.

- The analyses included adjustments for sociodemographics and health behaviours.

- Due to the cross-sectional design, temporal order is unknown and reverse causation may be present.

- Assessment was solely based on self-report.

A curvilinear relationship between depression and alcohol use indicating a higher depression risk for both abstinence and heavy drinking compared with a moderate consumption has been revealed by several recent cross-sectional and longitudinal studies. ${ }^{23}$ A considerable number of studies found curvilinear associations even after controlling for confounders such as drinking history, sociodemographics and healthrelated variables. ${ }^{45}$

Four limitations of the previous research on the association between depression and alcohol use exist. First, although medical care provides a valuable setting for managing depressive symptoms and alcohol use, given the frequent presentation of both conditions because of accompanying physical health problems, ${ }^{6} 7$ studies on the association of depression and alcohol use within medical care are scarce. Second, studies have been restricted to one measure of alcohol use, mainly average volume of intake. ${ }^{45}$ Only recently, more attention has been paid on consumption per occasion and excessive consumption days, and there is evidence that 
multiple measures have to be considered to properly capture differential alcohol use patterns. ${ }^{68}$ Third, categorically defined alcohol consumption groups were used, such as abstainers and low, moderate and heavy drinking individuals. Information might be lost by that in statistical testing. ${ }^{9}$ Previous studies did not analyse the association between depression and alcohol use over the full range on both scales. Only a few studies analysed continuous alcohol use measures, but specific functional forms were assumed (eg, quadratic or cubic). ${ }^{81011}$ Inherent in this third limitation is that results of studies may differ by applied cut-offs. Considering the full range of depressive symptom severity is particularly important in light of the high prevalence of subthreshold depression: depressive symptoms not meeting criteria for major depression. ${ }^{12}$ Fourth, there is a lack of understanding whether the same shape of association exists across gender and age. A substantial number of studies were restricted to agespecfic or gender-specific subpopulations. ${ }^{13} 14$ The few studies that exist found either more evidence for curvilinear associations in men or in women ${ }^{211} 15$ or suggest the association may change with age. ${ }^{11} 13$ As there is evidence that the nature of the interaction may depend on the measures used, ${ }^{8}$ a closer examination of the full continuum of different alcohol measures and their potential interactions with gender and age may elucidate inconsistencies from previous research.

One solution of the limitations might be to use the multivariable fractional polynomial (MFP) approach. ${ }^{16}$ It permits a systematically data-driven selection of the best-fitting functional form. Although it has been applied effectively in other research areas, ${ }^{17}$ no previous study has used MFP to test for curvilinearity in the association of depression and alcohol use so far.

\section{Aims}

The aims of the current study were (1) to predict depressive symptom severity and presence of major depression in medical care patients with respect to four continuous measures of alcohol use, (2) to determine the best-fitting functional form of these relationships and (3) to test whether these relationships are consistent across gender and age.

\section{METHODS}

\section{Patient and public involvement}

Patients and the public were not involved in the design or planning of the study.

\section{Sample}

Participants were men and women aged 18-64 years, recruited from 39 ambulatory practices and 56 hospital wards at three sites in Germany. From January 2017 to March 2018, consecutive patients were proactively approached by study assistants for an anonymous computerised screening on health risk factors. Verbal informed consent was obtained for participation in the screening.
Details of recruitment were reported elsewhere. ${ }^{18}$ Among all eligible patients, $13763(86.5 \%)$ were screened and 12828 completed the screening. Two individuals had to be excluded from data analysis because of missing data on relevant covariates. The final sample analysed comprised 12826 patients.

\section{Measures}

Two depression measures, depressive symptom severity and presence of major depression, were assessed. In the first step, three questions derived from the German version of the Diagnostic and Statistical Manual of Mental Disorders, Fourth Edition (DSM-IV) Composite International Diagnostic Interview (CIDI) ${ }^{19}$ were presented: 'In the past 12 months, have you had a period of 2 weeks or longer when you ... "(a) "felt sad, despondent, or depressed almost daily for most of the time?", (b) "lacked energy or felt tired or exhausted all the time, even when you had not been doing hard work or have been physically ill?", and (c) "lost interest in most things? This means, for example, hobbies, leisure, and being together with friends, that is things you usually enjoy?'. Participants endorsing any of the three questions received additionally the eight-item version of the Patient Health Questionnaire (PHQ-8), ${ }^{20}$ asking for symptoms during their most severe episode of depression within the past 12 months. This self-report instrument assesses eight of the nine DSM-IV criteria for major depression (excluding suicidal thoughts or actions) on a 4-point Likert scale ( 0 , 'not at all'; 1 , 'on several days'; 2, 'more than half of the days'; and 3, 'nearly every day"). The PHQ-8 sum score was calculated to represent symptom severity (range 0-24). Participants endorsing none of the three screening questions were coded with a PHQ-8 sum score of 0 . The presence of major depression was defined as endorsing at least one of the three CIDI screening questions and reaching a PHQ-8 sum score of $\geq 10$. This cut-off was chosen based on previous validation studies of Kroenke and Spitzer, ${ }^{21}$ who showed that within a large sample of primary care patients, a cutoff of $\geq 10$ yielded high sensitivity ( $\geq 99 \%$ ) and specificity (91\%-92\%) for diagnosing major depression when using a blinded mental health professional structured interview as gold standard.

Four alcohol use measures were assessed based on an expanded version of the Alcohol Use Disorder Identification Test (AUDIT) ${ }^{1822}$ : alcohol consumption per day, alcohol consumption per occasion, number of excessive consumption days per month and the AUDIT sum score: (1) a continuously assessed number of drinks consumed per occasion were multiplied by $10.5 \mathrm{~g}$ to calculate the average alcohol consumption in grams per occasion; (2) answers for drinking occasions were given as never, once a month or less, twice, three or four times a month, and twice, three, four, five, six or seven times a week, and were converted into a continuous measure by using the corresponding values describing the monthly frequency; average alcohol consumption in grams per day was calculated as drinking occasions per month times average 
alcohol consumption in grams per occasion divided by 28; (3) excessive consumption frequency was assessed for men and women separately with different thresholds ${ }^{23}$ : 'How often do you drink five or more alcoholic drinks on one occasion?' and 'How often do you drink four or more alcoholic drinks on one occasion?' Answers given (never, less than once, once, twice, three, four times a month; twice, three, four, five, six, seven times a week) were converted into a continuous measure by using the corresponding values describing the monthly frequency; and (4) based on the original items, an AUDIT sum score was calculated ranging from 0 to 40 . Resulting scale values for all alcohol use measures can be found in online supplementary tables S1-S4.

Potential confounders were assessed as described here ${ }^{18}$ and included (1) sociodemographics such as recruitment setting (inpatients and outpatients), recruitment site (sites 1, 2 and 3), gender, age (continuous), family status (married/in partnership, single/divorced/widowed), educational level (less than 10, 10 or more than 10 years) and occupational status (full-time, part-time employed, unemployed or not working, eg, home maker, retiree, student or similar); and (2) health-related variables such as general health status (continuous and recalibrated $^{24}{ }^{25}$ ), smoking status (current daily, current less than daily, former and never smoker), number of fruit and vegetable servings consumed per week ${ }^{26}$ (continuous), total minutes of leisure-time physical activity per week ${ }^{27}$ (continuous) and Body Mass Index (BMI) (continuous).

\section{Statistical analysis}

All analyses were performed using STATA V.14.2. First, descriptive statistics were reported of the sample characteristics stratified by daily alcohol intake categories $(0,<1,1-5,>5-15$ and $>15 \mathrm{~g})$. Continuous variables were expressed as mean and SD or median and IQR in case of skewed distributions. Categorical variables were expressed as absolute and percent values.

We predicted depressive symptom severity (using negative binomial regression) and presence of major depression (using logistic regression) by each of the four alcohol use measures (alcohol consumption per day, alcohol consumption per occasion, number of excessive consumption days per month and the AUDIT sum score). Two-step adjustment was introduced for all regression models: (1) sociodemographic-adjusted models and (2) multiple-adjusted models, including additionally healthrelated variables. Rate ratios and ORs, along with 95\% CIs, were calculated.

Within statistical modelling (eg, logistic regression), a continuous covariate $\mathrm{X}$ is traditionally assumed to be linearly associated with the response variable $\mathrm{Y}$, resulting in a straight regression line with $\mathrm{Y}=\beta_{0}+\beta_{1} X$. However, this assumption of linearity may be incorrect. Fractional polynomials (FP) ${ }^{16}$ avoid this assumption and permit modelling of curvilinear associations. FPs are extensions of power transformations and systematically investigate which transformation of a continuous covariate X may fit the data best. FP functions of firstdegree fit one power transformation of the form $\mathrm{X}^{\mathrm{P}}$, whereas FP functions of second-degree fit two power transformations of the form $\mathrm{X}^{\mathrm{P}}$, resulting in regression equations of the form $\mathrm{Y}=\beta_{0}+\beta_{1} \mathrm{X}^{\mathrm{P}}$ and $Y=\beta_{0}+\beta_{1} \mathrm{X}^{\mathrm{P}}+\beta_{2} \mathrm{X}^{\mathrm{P}}$, respectively. Different exponents are fitted successively to determine the best $\mathrm{FP}$ function. By default $\mathrm{P}$ is chosen from a small set of $\{-2,-1,-0.5,0,0.5,1,2,3\}$ where $x^{0}$ denotes $\ln (x)$. In the first step, the best FP function of first degree and the best FP function of second degree are determined based on the model deviance (minus twice the maximised $\log$ likelihood). In the second step, a closed test procedure systematically tests whether a linear function or an FP function of first degree is sufficient to describe the shape of the association between $\mathrm{X}$ and $\mathrm{Y}$, or whether a more complex FP function of second degree is needed. The closed test procedure ensures that the overall type 1 error for selecting an FP function is close to our nominal significance level of .05. An example of FP function selection can be found in online supplementary file 1 . To explore curvilinear associations when predicting depressive symptom severity and presence of major depression by alcohol use, $\mathrm{MFP}^{16}$ was applied, which investigates FP functions simultaneously for all continuous covariates. Automatic scaling and centreing were performed during the selection process to improve numerical stability. Graphical presentation of the curvilinear associations within the multiple-adjusted models was generated..$^{28}$ Therefore, rate ratios and ORs were calculated to a reference level of the alcohol use measures, which was chosen according to the lowest predicted depressive symptom severity or odds of major depression, respectively. ${ }^{4}$

Within the multiple-adjusted models, the two-way interaction with gender and age was tested for each alcohol use measure. ${ }^{29}$ Significant interactions ( $p$ value of $<0.05$ according to the likelihood ratio test) were then graphically displayed as mean predicted depressive symptom severity and probability of major depression by gender and age percentiles.

MFP model evaluation was performed by graphical comparison of the fully adjusted main MFP models with the same MFP models (1) omitting subject with extreme values $(>80 \mathrm{~g}$ alcohol per day, $>300 \mathrm{~g}$ alcohol per occasion) and (2) with a constant for 0 values, assuming the effect of alcohol use measures may not be continuous between 0 and the next higher values. ${ }^{30}$ Significant interactions found within the MFP models were graphically verified using running line smoothed locally weighted scatterplot smoothing (LOWESS) regressions on raw data in each gender and four equalsized age groups. ${ }^{16}$

\section{RESULTS}

Participants' characteristics are presented in table 1 . The average gram per day consumed in the total sample was $3.7(\mathrm{SD}=9.0)$, with a median of $1.1(\mathrm{IQR}=0.4-3.4)$. 


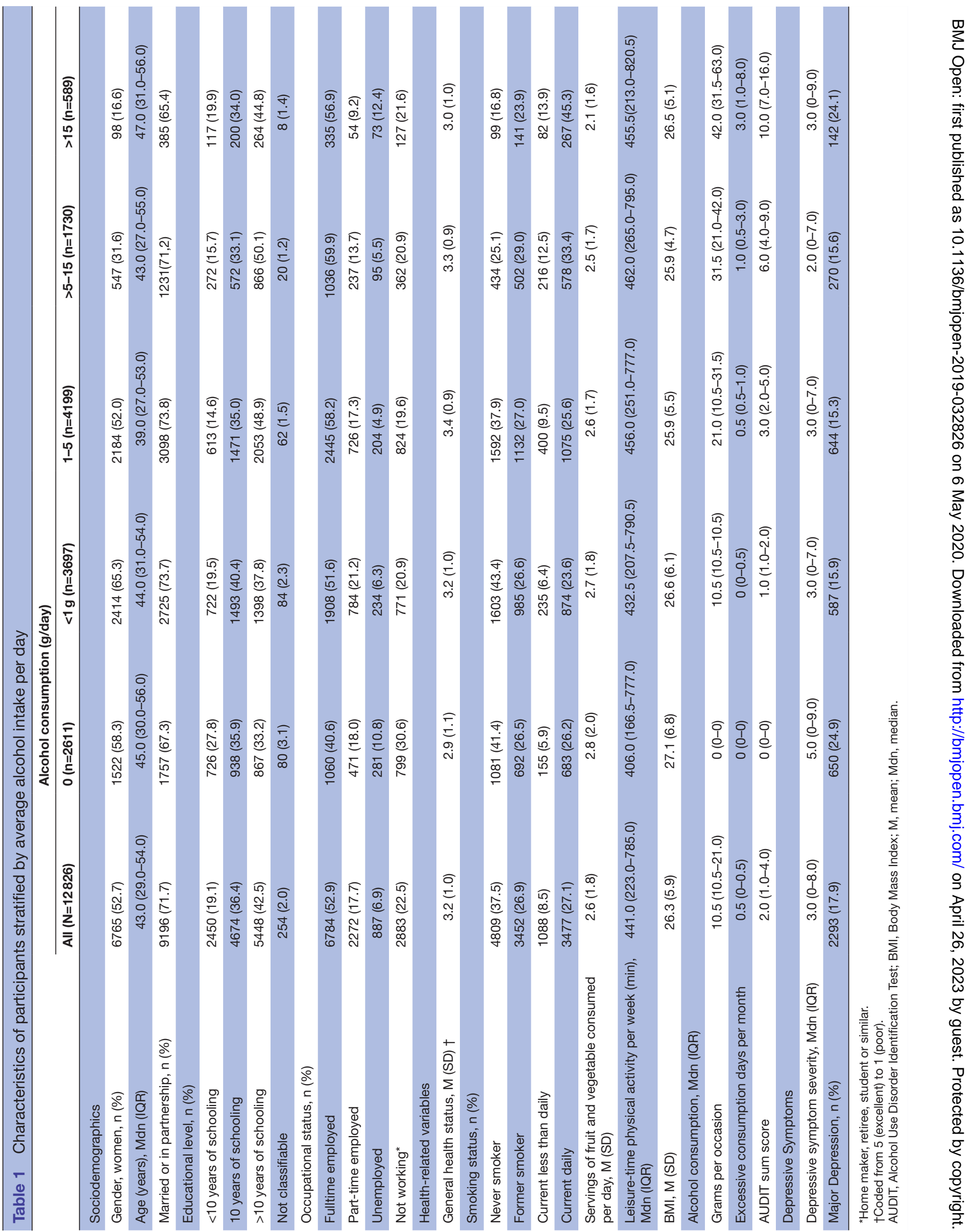


Table 2 Results of negative binomial regression analyses to predict depressive symptom severity by alcohol use measures

\begin{tabular}{|c|c|c|c|c|}
\hline \multirow[b]{2}{*}{ Independent variables } & \multicolumn{2}{|c|}{ Sociodemographic-adjusted* } & \multicolumn{2}{|c|}{ Multiple-adjusted $†$} \\
\hline & Power & Rate ratio $(95 \% \mathrm{Cl})$ & Power & Rate ratio $(95 \% \mathrm{Cl})$ \\
\hline Alcohol (g/day) & -2 & 1.000005 (1.000004 to 1.000006$)$ & -2 & $1.000003(1.000002$ to 1.000005$)$ \\
\hline \multirow[t]{2}{*}{ Alcohol (g/occasion) } & -2 & 1.01 (1.01 to 1.01$)$ & -2 & 1.01 (1.01 to 1.01$)$ \\
\hline & -0.5 & 0.60 (0.52 to 0.70$)$ & -0.5 & 0.70 (0.61 to 0.80$)$ \\
\hline \multirow[t]{2}{*}{ AUDIT } & 0 & 1.67 (1.52 to 1.84$)$ & 0 & 1.50 (1.37 to 1.64$)$ \\
\hline & 0 & 1.28 (1.23 to 1.33$)$ & 0 & $1.20(1.16$ to 1.25$)$ \\
\hline
\end{tabular}

Power indicates powers of the FPs used to model the independent variables, for example, in the sociodemographics-adjusted model alcohol g/day is modelled by $\beta_{1} \times$ alcohol $\mathrm{g} /$ day $^{-2}+\beta_{2} \times$ alcohol $\mathrm{g} /$ day $^{1}$, where $\beta_{\mathrm{n}}$ is the associated unexponentiated regression coefficient.

All alcohol use measures were significant with $p<0.001$.

*Adjusted for recruitment setting, recruitment site, gender, age (modelled as a FP function of second degree with powers (3 3)), family status, educational level and occupational status.

†Adjusted for recruitment setting, recruitment site, gender, age (modelled as a FP function of second degree with powers (2 3)), family status, educational level, occupational status, general health status, smoking status, fruit and vegetable consumption, leisure-time physical activity and Body Mass Index.

AUDIT, Alcohol Use Disorder Identification Test; FP, fractional polynomial.

For all alcohol use measures, FP functions of second degree were applied as these significantly improved model fit compared with less complex FP functions of first degree and linear models according to the closed test procedure (see tables 2 and 3, column Power). Within the continuous confounders included, only age showed significant deviations from linearity, and FP transformation of second degree was applied (see tables 2 and 3 , legend). General health status, fruit and vegetable consumption, leisure-time physical activity and BMI were modelled as linear.

The negative binomial and logistic regression analyses showed consistent significant curvilinear associations when predicting depressive symptom severity (table 2) and presence of major depression (table 3) by the alcohol use measures and adjusting for sociodemographics. Those with an average consumption of $1.1 \mathrm{~g} /$ day, $10.5 \mathrm{~g} /$ occasion, one excessive consumption day per month and those with an AUDIT score of 2 had the lowest predicted depressive symptom severity and odds of major depression (online supplementary tables S1-S4, reference). Observed values below these reference levels were associated with higher predicted depressive symptom severity and higher odds of major depression. For values above the reference levels, the predicted depressive symptom severity and odds of major depression were increasing for all alcohol measures.

Table 3 Results of logistic regression analyses to predict the presence of major depression by alcohol use measures

\begin{tabular}{|c|c|c|c|c|}
\hline \multirow[b]{2}{*}{ Independent variables } & \multicolumn{2}{|c|}{ Sociodemographic-adjusted* } & \multicolumn{2}{|c|}{ Multiple-adjusted $†$} \\
\hline & Power & OR (95\% Cl) & Power & OR (95\% Cl) \\
\hline Alcohol (g/day) & -2 & $1.00001(1.000008$ to 1.000012$)$ & -2 & 1.000007 (1.000005 to 1.000009$)$ \\
\hline \multirow[t]{2}{*}{ Alcohol (g/occasion) } & -2 & $1.02(1.02,1.03)$ & -2 & 1.03 (1.02 to 1.04$)$ \\
\hline & -0.5 & 0.35 (0.27 to 0.44$)$ & -1 & 0.74 (0.67 to 0.82$)$ \\
\hline Excessive consumption (days/month) & 0 & 1.19 (1.14 to 1.25$)$ & 0.5 & 3.08 (2.00 to 4.77$)$ \\
\hline \multirow[t]{2}{*}{ AUDIT } & -2 & 1.01 (1.01 to 1.01$)$ & -1 & 1.10 (1.08 to 1.13$)$ \\
\hline & 1 & 2.46 (2.16 to 2.80$)$ & 1 & 2.47 (2.12 to 2.88$)$ \\
\hline
\end{tabular}

Power indicates powers of the FPs used to model the independent variables, for example, in the sociodemographics-adjusted model alcohol g/day is modelled by $\beta_{1} \times$ alcohol $\mathrm{g} / \mathrm{day}^{-2}+\beta_{2} \mathrm{x}$ alcohol $\mathrm{g} / \mathrm{day}^{0.5}$, where $\beta_{\mathrm{n}}$ is the associated unexponentiated regression coefficient.

All alcohol use measures were significant with $p<0.001$.

*Adjusted for recruitment setting, recruitment site, gender, age (modelled as a FP function of second degree with powers (3 3)), family status, educational level and occupational status.

†Adjusted for recruitment setting, recruitment site, gender, age (modelled as a FP function of second degree with powers (2 3)), family status, educational level, occupational status, general health status, smoking status, fruit and vegetable consumption, leisure-time physical activity and Body Mass Index.

AUDIT, Alcohol Use Disorder Identification Test; FP, fractional polynomial. 
(a)

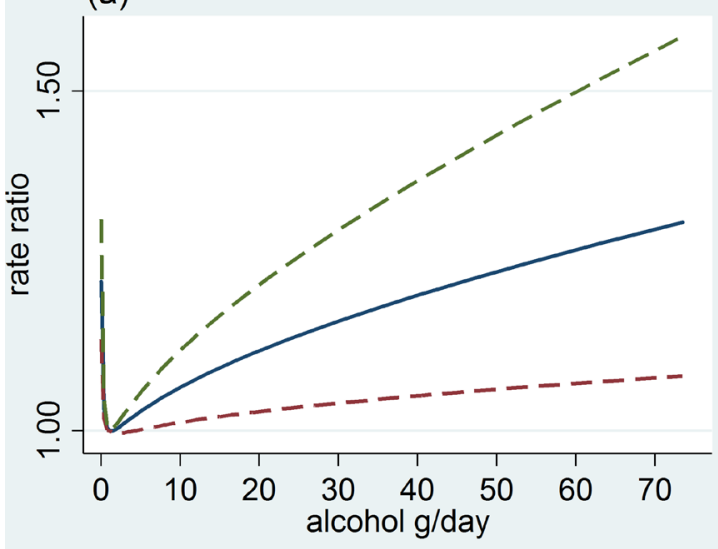

(c)

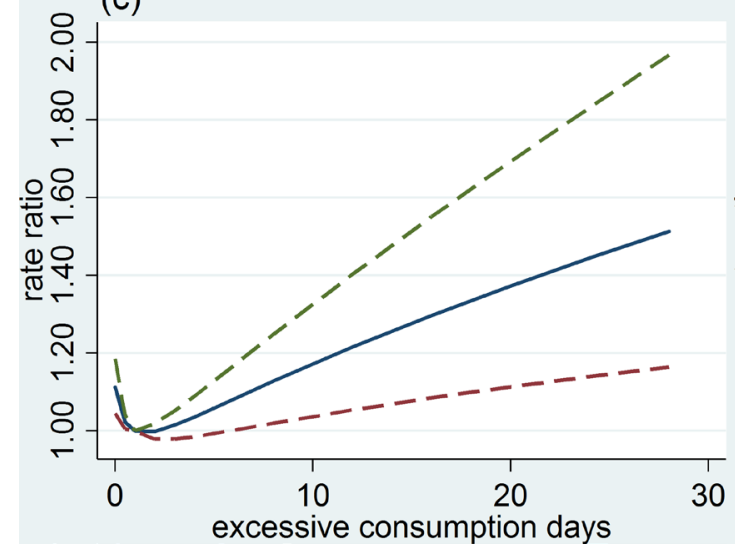

(b)

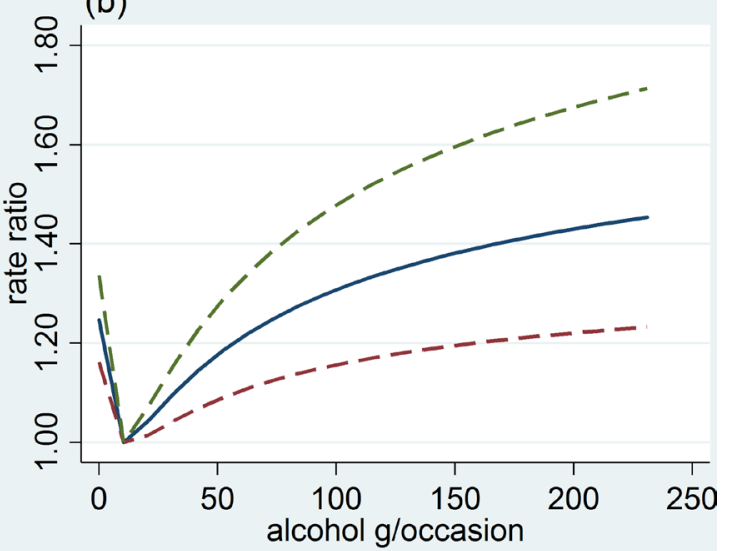

(d)

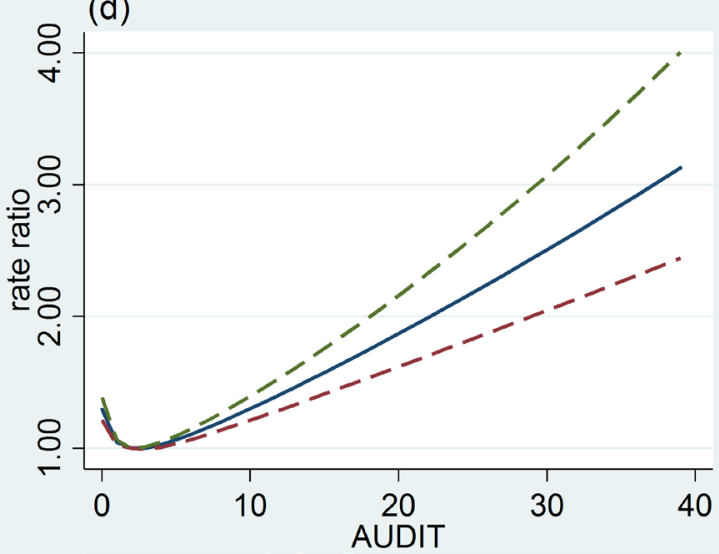

Figure 1 Prediction of depressive symptom severity by four alcohol use measures (multiple-adjusted). Note: associations of depressive symptom severity with alcohol g/day (A), alcohol g/occasion (B), excessive consumption days (C) and AUDIT sum score (D) were estimated by multivariable fractional polynomial approach using negative binomial regression analyses adjusted for recruitment setting, recruitment site, gender, age, family status, educational level, occupational status, general health status, smoking status, fruit and vegetable consumption, leisure-time physical activity and Body Mass Index. Displayed are rate ratios with $95 \%$ Cls calculated to the following reference levels: alcohol g/day $=1.125 \mathrm{~g}$, alcohol g/occasion $=10.5 \mathrm{~g}$, excessive consumption days/month=1, AUDIT sum score=2. For readability reasons, graphics for alcohol g/day were restricted to a consumption of no more than $80 \mathrm{~g}(99.8 \%$ percentile) and for alcohol g/occasion, to a consumption of no more than $300 \mathrm{~g}$ (99.98\% percentile). AUDIT, Alcohol Use Disorder Identification Test.

Health-related variables could not fully explain the curvilinear associations, as they remained significant in the multiple-adjusted models (second column of tables 2 and 3). Although this effect was attenuated, values of the alcohol measures below the reference levels were still significantly associated with higher predicted depressive symptom severity and odds of major depression compared with the reference levels (online supplementary tables S1-S4). Multiple-adjusted models are displayed in figures 1 and 2. Rate ratios and ORs for the prediction of depressive symptom severity and presence of major depression aligning to each observed value of the alcohol use measures are shown in online supplementary tables S1-S4. Model evaluation analyses confirmed the basic shapes of main associations across alcohol use measures (online supplementary figure S1). Omitting subjects with extreme values or adding a constant for zero values (assuming abstainers may be qualitatively different from drinkers) affected estimates mainly at the upper end of the alcohol use scales; for example, omitting subjects with extreme values resulted in higher depressive symptom severity estimates for daily consumptions above 25 g compared with the main models.

We found significant interactions of gender with all alcohol use measures when predicting depressive symptom severity and presence of major depression, and higher depression outcomes for women compared with men (online supplementary figures S2 and S3). Most of women's risk curves showed shallower decreases on the left tail and steeper increases at the right tail of the alcohol distribution compared with men's risk curves. All main predictions of depressive symptom severity and presence of major depression by the alcohol use measures were still significant after introducing interaction with gender (not shown), except for the prediction of depressive symptom severity by excessive consumption days per month as this was only significant among men $(\mathrm{p}<0.001)$ but not among women $(\mathrm{p}=0.75)$.

Age significantly modified the prediction of depressive symptom severity by excessive consumption days per month, as well as the prediction of presence of major depression by all alcohol use measures with higher 
(a)

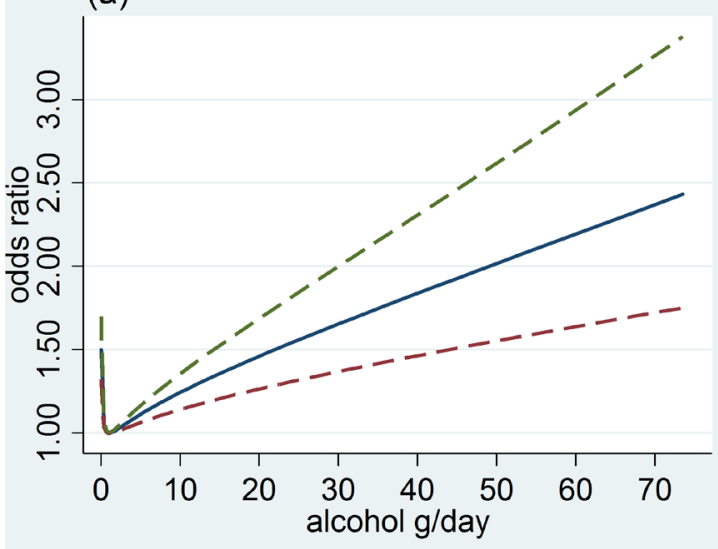

(c)

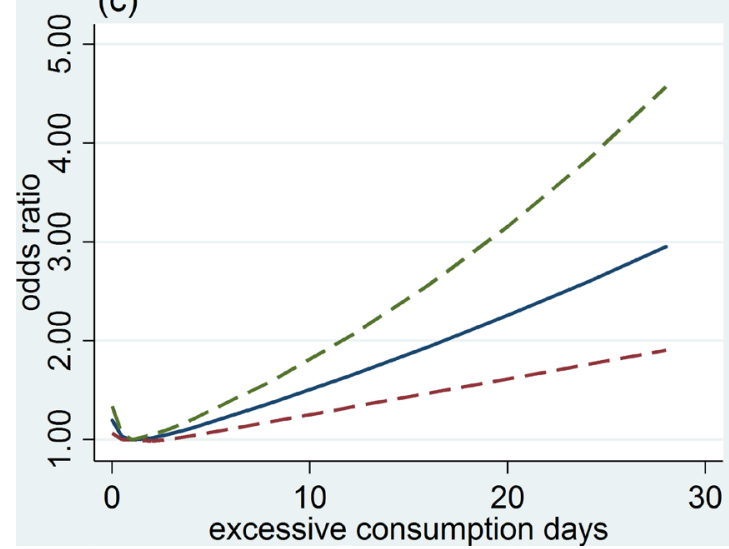

(b)

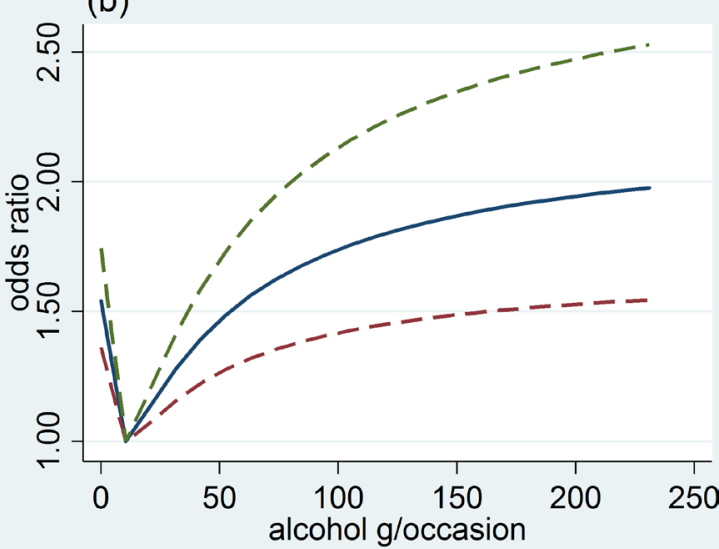

(d)

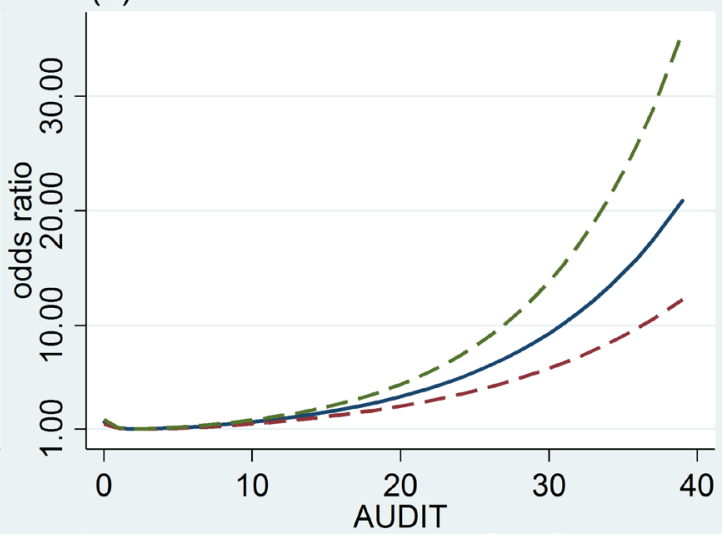

Figure 2 Prediction of major depression by four alcohol use measures (multiple-adjusted). Note: associations of major depression with alcohol g/day (A), alcohol g/occasion (B), excessive consumption days (C) and AUDIT sum score (D) were estimated by multivariable fractional polynomial approach using logistic regression analyses adjusted for recruitment setting, recruitment site, gender, age, family status, educational level, occupational status, general health status, smoking status, fruit and vegetable consumption, leisure-time physical activity and Body Mass Index. Displayed are ORs with 95\% Cls calculated to the following reference levels: alcohol $\mathrm{g} / \mathrm{day}=1.125 \mathrm{~g}$, alcohol $\mathrm{g} / \mathrm{occasion}=10.5 \mathrm{~g}$, excessive consumption days $/ \mathrm{month}=1$, AUDIT sum score=2. For readability reasons, graphics for alcohol g/day were restricted to a consumption of no more than $80 \mathrm{~g}$ (99.8\% percentile) and for alcohol g/occasion, to a consumption of no more than $300 \mathrm{~g}$ (99.98\% percentile). AUDIT, Alcohol Use Disorder Identification Test.

depression outcomes for younger individuals (online supplementary figures S2 and S3). For most of these risk curves, younger individuals showed shallower decreases on the left tail and steeper increases on the right tail of the alcohol distribution compared with older individuals. For excessive consumption days per month, the graphics show that the effect approximates to a more linear prediction for those at the lowest age percentile. When predicting depressive symptom severity, there was no significant interaction between age and alcohol consumption per day $\left(\mathrm{X}^{2}=5.8, \mathrm{p}=0.05\right)$, alcohol consumption per occasion $\left(\mathrm{X}^{2}=2.2, \mathrm{p}=0.33\right)$ and AUDIT $\left(\mathrm{X}^{2}=3.6, \mathrm{p}=0.16\right)$. Slope differences within smoothed LOESS regression across gender and age verified interactions identified with MFP models (online supplementary figure S4).

\section{DISCUSSION}

This study revealed that alcohol use and depression in medical care patients are associated in a curvilinear manner. This curvilinear relationship was evident (1) for both depressive symptom severity and presence of major depression, (2) for four continuous alcohol use measures, (3) after controlling for sociodemographics and health behaviours, and (4) across gender and age with varying steepness of the risk slopes.

For three of four alcohol use measures used (consumption per day, consumption per occasion and the AUDIT score) significantly increased depressive symptom severity, and odds of major depression were already evident below the established limits for low-risk drinking. ${ }^{22} 23$

Comparisons with previous findings are difficult due to the limitations we have reported. However, with respect to consumption per occasion and excessive consumption days per month, our results were in line with results from a large general population sample. ${ }^{8}$ Our findings for the AUDIT were also in line with two previous studies suggesting lowest depression levels at an AUDIT score of four in an Australian general population sample ${ }^{11}$ and within the AUDIT categories 2-4 in a medical care sample across 14 countries. ${ }^{3}$ 
We found that the association of depressive symptom severity and presence of major depression with alcohol use differs between women and men. This may serve as an explanation for previous inconsistent research, which sometimes found weaker or no associations for women. ${ }^{2}{ }^{25}$ Differences by gender may be reasonable, considering differences in drinking patterns ${ }^{31}$ and differences in the expression of depressive symptoms. ${ }^{32}$ The fact that the steepness of the initial decrease of depression risk increased by age may be due to the worsening health status associated with increasing age which makes effects of sick quitting operate more strongly. ${ }^{33}$ Furthermore, medication use can be expected to increase with age, and reduction of alcohol consumption may be therefore due to avoidance of interactions with prescribed medication. ${ }^{34}$

\section{Strengths and limitations}

Strengths of the study include the large and proactively recruited medical care sample and a high proportion of participants reached among all patients approached. Proactive recruitment may have reduced selection bias compared with previous studies. ${ }^{35}$ This is the first study that conducted MFP on continuous independent variables to systematically model curvilinearity in the association of depression and alcohol use, which offers more flexibility in the shape of the association than just adding quadratic or cubic terms ${ }^{16}$ and uses full information in contrast to a priori categorisation. ${ }^{9}$ Lastly, we statistically tested for gender and age interaction within the MFP approach rather than relying on subgroup analyses.

Our findings should be interpreted in the context of the following limitations: first, due to the cross-sectional nature of our study, the temporal order is unknown and reverse causation ${ }^{36}$ may be present. However, longitudinal studies including only participants without the presence of depression at baseline confirmed curvilinear associations of incidence of depression and alcohol use. ${ }^{5}$ It seems likely that associations between depression and alcohol use are reciprocal, and it has been suggested that the dominant underlying causal process may be different, depending on the severity of alcohol use. ${ }^{37}$ Second, we did not assess lifetime history of depression and drinking. Therefore, we cannot exclude that previous depressioninduced or alcohol-induced health issues led to abstinence or reduced drinking in some individuals. ${ }^{33}$ Given the dynamic nature of alcohol consumption, current abstainers in our study may consist of lifetime abstainers and former drinkers, and current non-abstainers consist of reducers and non-reducers, which may produce relatively lower-risk differences across the full continua of alcohol consumption. ${ }^{15}$ In the same way, individuals were mixed with respect to their history of depressive symptoms, including those who had never experienced depressive symptoms and those with first onset of depression or recurrent depressive symptoms. Third, other potential relevant variables such as social factors, ${ }^{4}$ physical or mental health, ${ }^{10}$ medication, ${ }^{34}$ drinking motives ${ }^{38}$ or preferred beverage ${ }^{5}$ have not been included in the present study. This is important as abstainers may feature pre-existing characteristics that put them at higher risk of depression and/or lead them to choose to abstain. ${ }^{39}$ Fourth, assessment was solely based on self-report. Fifth, patients endorsing none of the three depression screening questions were coded with a PHQ-8 sum score of 0 . However, it is likely that at least some of those would have reached a PHQ-8 sum score above 0 if the PHQ-8 was provided. Thus, depressive symptom severity and prevalence of major depression may have been underestimated in our sample.

\section{CONCLUSIONS}

The findings of this study indicate that alcohol use and depression in medical care patients are associated in a curvilinear manner. Depressive symptom severity and presence of major depression increased from very low to high alcohol consumption levels and were also higher in abstinent patients across all four alcohol use measures. Found associations differed by gender and age, with higher depression outcomes along the full continuum of alcohol use and steeper risk increases in women and younger individuals for most alcohol use measures. However, caution is needed in the interpretation of these results due to the cross-sectional design and constraints in data collection. Cohort studies are needed to examine causal associations between depression and alcohol use.

\section{Author affiliations}

${ }^{1}$ Institute for Community Medicine, Department of Social Medicine and Prevention, University Medicine Greifswald, Greifswald, Germany

${ }^{2}$ DZHK (German Center for Cardiovascular Research), Partner Site Greifswald, Greifswald, Germany

${ }^{3}$ Department of Psychiatry and Psychotherapy, University Hospital of Tübingen, Tübingen, Germany

${ }^{4}$ Institute of Medical Psychology, University Medicine Greifswald, Greifswald, Germany

${ }^{5}$ Department of Psychiatry and Psychotherapy, University of Lübeck, Lübeck, Germany

Contributors UJ, CM, AB, JF-A and SU outlined the concepts of the underlying studies in the Addiction: Early Recognition and Intervention Across the Lifespan grant proposal. DG, AM, KK, AB, SE, H-JR, GB and CM were responsible for organising the fieldwork. DG, AM, KK, CM and SE were responsible for preparation and checks of the data. DG and CM developed the initial concept of the current study. DG undertook the analysis with input and advice from all coauthors. DG drafted the initial version of the manuscript. All authors revised the manuscript for important intellectual content and read and approved the final manuscript.

Funding This study is embedded in the research consortium Addiction: Early Recognition and Intervention Across the Lifespan, funded by the German Federal Ministry of Education and Research (BMBF) within the Research Network on Mental Disorders (grant numbers 01EE1406F, 01EE1406E and 01EE1406H). We acknowledge support for the Article Processing Charge from the DFG (German Research Foundation, 393148499) and the Open Access Publication Fund of the University of Greifswald, Germany.

Competing interests None declared.

Patient consent for publication Not required.

Ethics approval Ethical approvals were obtained from the institutional review boards of the participating sites (ethical review committee of the Faculty of Medicine, University Medicine Greifswald: BB 170/16, BB 161/16; ethical review committee of the Faculty of Medicine, University Hospital Tübingen: 598/2016B02; 
ethical review committee of the Faculty of Medicine, University of Lübeck: 15-256) and conformed to the principles set out in the Declaration of Helsinki.

Provenance and peer review Not commissioned; externally peer reviewed.

Data availability statement Data are available upon reasonable request.

Open access This is an open access article distributed in accordance with the Creative Commons Attribution Non Commercial (CC BY-NC 4.0) license, which permits others to distribute, remix, adapt, build upon this work non-commercially, and license their derivative works on different terms, provided the original work is properly cited, appropriate credit is given, any changes made indicated, and the use is non-commercial. See: http://creativecommons.org/licenses/by-nc/4.0/.

ORCID iD

Diana Guertler http://orcid.org/0000-0002-2533-5347

\section{REFERENCES}

1 Riper H, Andersson G, Hunter SB, et al. Treatment of comorbid alcohol use disorders and depression with cognitive-behavioural therapy and motivational interviewing: a meta-analysis. Addiction 2014;109:394-406.

2 Bell S, Britton A, Kubinova R, et al. Drinking pattern, abstention and problem drinking as risk factors for depressive symptoms: evidence from three urban eastern European populations. PLoS One 2014:9:e104384.

3 Bellos S, Skapinakis P, Rai D, et al. Longitudinal association between different levels of alcohol consumption and a new onset of depression and generalized anxiety disorder: results from an international study in primary care. Psychiatry Res 2016;243:30-4.

4 Skogen JC, Harvey SB, Henderson M, et al. Anxiety and depression among abstainers and low-level alcohol consumers. The NordTrøndelag health study. Addiction 2009;104:1519-29.

5 Gea A, Beunza JJ, Estruch R, et al. Alcohol intake, wine consumption and the development of depression: the PREDIMED study. BMC Med 2013;11:192.

6 Rehm J, Gmel GE, Gmel G, et al. The relationship between different dimensions of alcohol use and the burden of disease-an update. Addiction 2017;112:968-1001.

7 Kroenke K. Patients presenting with somatic complaints: epidemiology, psychiatric comorbidity and management. Int $J$ Methods Psychiatr Res 2003;12:34-43.

8 Graham K, Massak A, Demers A, et al. Does the association between alcohol consumption and depression depend on how they are measured? Alcohol Clin Exp Res 2007;31:78-88.

9 Royston P, Altman DG, Sauerbrei W. Dichotomizing continuous predictors in multiple regression: a bad idea. Stat Med 2006;25:127-41.

10 Kim S-A, Kim E, Morris RG, et al. Exploring the non-linear relationship between alcohol consumption and depression in an elderly population in Gangneung: the Gangneung health study. Yonsei Med J 2015;56:418-25.

11 Rodgers B, Korten AE, Jorm AF, et al. Non-Linear relationships in associations of depression and anxiety with alcohol use. Psychol Med 2000;30:421-32.

12 Rucci P, Gherardi S, Tansella M, et al. Subthreshold psychiatric disorders in primary care: prevalence and associated characteristics. $J$ Affect Disord 2003;76:171-81.

13 Alati R, Lawlor DA, Najman JM, et al. Is there really a 'J-shaped' curve in the association between alcohol consumption and symptoms of depression and anxiety? Findings from the MaterUniversity Study of Pregnancy and its outcomes. Addiction 2005;100:643-51.

14 Kirchner JE, Zubritsky C, Cody M, et al. Alcohol consumption among older adults in primary care. J Gen Intern Med 2007;22:92-7.

15 Caldwell TM, Rodgers B, Jorm AF, et al. Patterns of association between alcohol consumption and symptoms of depression and anxiety in young adults. Addiction 2002;97:583-94.

16 Royston P, Sauerbrei W. Multivariable Model-Building: A Pragmatic Approach to Regression Analysis based on Fractional Polynomials for Modelling Continuous Variables. West Sussex: JohnWiley \& Sons Ltd, 2008.
17 Cao S, Moineddin R, Urquia ML, et al. J-shapedness: an often missed, often miscalculated relation: the example of weight and mortality. J Epidemiol Community Health 2014;68:683-90.

18 Guertler D, Moehring A, Krause K, et al. Proactive multipurpose health risk screening in health care settings: methods, design, and reach. Int J Methods Psychiatr Res 2019;28:p. e1760.

19 WittchenHet al. Munich composite international diagnostic interview (M-CIDI). MaxPlanck-Institut für Psychiatrie: München 1995.

20 Kroenke K, Strine TW, Spitzer RL, et al. The PHQ-8 as a measure of current depression in the general population. J Affect Disord 2009;114:163-73.

21 Kroenke K, Spitzer RL. The PHQ-9: A new depression diagnostic and severity measure. Psychiatr Ann 2002;32:509-15.

22 Saunders JB, Aasland OG, Babor TF, et al. Development of the Alcohol Use Disorders Identification Test (AUDIT): WHO Collaborative Project on Early Detection of Persons with Harmful Alcohol Consumption--II. Addiction 1993;88:791-804.

23 National Institute of alcohol abuse and alcoholism. drinking levels defined., 2012. Available: https://www.niaaa.nih.gov/alcohol-health/ overview-alcohol-consumption/moderate-binge-drinking [Accessed 201819 Feb]

24 McHorney CA, Ware JE, Raczek AE. The mos 36-Item short-form health survey (SF-36): II. psychometric and clinical tests of validity in measuring physical and mental health constructs. Med Care 1993;31:247-63.

25 Ware J. Sf-36 health survey manual and interpretation guide. The health Institute, 1993. Available: http://czresearch.com/info/SF36_ healthsurvey_ch6.pdf [Accessed 20187 June].

$26 \mathrm{WHO}$. The who stepwise approach to noncommunicable disease risk factor surveillance (steps) Intrument. n.d. Available: http://www. who.int/chp/steps/instrument/STEPS_Instrument_V3.1.pdf?ua=1 [Accessed $20171 \mathrm{Dec}$ ].

27 Godin G. Commentary - The Godin-Shepard Leisure-Time Physical Activity Questionnaire. Health \& Fitness Journal of Canada 2011;4:18-22.

28 Ittermann T. EPRESENT: Stata module to present non-linear relationships in regression models with log or logit-link. In: Statistical Software Components S458562, Boston College Department of Economics, 2018.

29 Royston P. MFPIGEN: Stata module for modelling and displaying interactions between continuous predictors. In: Statistical Software Components S457439, Boston College Department of Economics, 2012.

30 Royston P, Sauerbrei W, Becher H. Modelling continuous exposures with a 'spike' at zero: a new procedure based on fractional polynomials. Stat Med 2010;29:n/a-1227.

31 Brienza RS, Stein MD. Alcohol use disorders in primary care: do gender-specific differences exist? J Gen Intern Med 2002;17:387-97.

32 Cavanagh A, Wilson CJ, Kavanagh DJ, et al. Differences in the expression of symptoms in men versus women with depression: a systematic review and meta-analysis. Harv Rev Psychiatry 2017;25:29-38.

33 Fillmore KM, Stockwell T, Chikritzhs T, et al. Moderate alcohol use and reduced mortality risk: systematic error in prospective studies and new hypotheses. Ann Epidemiol 2007;17:S16-23.

34 Graham K, Massak A. Alcohol consumption and the use of antidepressants. CMAJ 2007;176:633-7.

35 Krause K, Guertler D, Moehring A, et al. Feasibility and acceptability of an intervention providing computer-generated tailored feedback to target alcohol consumption and depressive symptoms in Proactively recruited health care patients and Reactively recruited media volunteers: results of a pilot study. Eur Addict Res 2019;25:119-31.

36 Sullivan LE, Fiellin DA, O'Connor PG. The prevalence and impact of alcohol problems in major depression: a systematic review. Am J Med 2005;118:330-41.

37 Bell S, Britton A. Drinking pattern during midlife and risk of developing depression during 28 years of follow-up: a prospective cohort study. Drug Alcohol Depend 2015;155:111-7.

38 Wicki M, Kuntsche E, Eichenberger Y, et al. Different drinking motives, different adverse consequences? Evidence among adolescents from 10 European countries. Drug Alcohol Rev 2017;36:731-41.

39 Naimi TS, Stockwell T, Zhao J, et al. Selection biases in observational studies affect associations between 'moderate' alcohol consumption and mortality. Addiction 2017;112:207-14. 